\title{
Coordination of Distinct Motor Structures through Remote Axonal Coupling of Projection Interneurons
}

\author{
Jian Jing, Kosei Sasaki, Matthew H. Perkins, Michael J. Siniscalchi, Bjoern C. Ludwar, Elizabeth C. Cropper, \\ and Klaudiusz R. Weiss \\ Department of Neuroscience, Mount Sinai School of Medicine, New York, New York 10029
}

\begin{abstract}
Complex behaviors often require coordinated movements of dissimilar motor structures. The underlying neural mechanisms are poorly understood. We investigated cycle-by-cycle coordination of two dissimilar feeding structures in Aplysia californica: the external lips and the internal radula. During feeding, the lips open while the radula protracts. Lip and radula motoneurons are located in the cerebral and buccal ganglia, respectively, and radula motoneurons are controlled by a well characterized buccal central pattern generator (CPG). Here, we examined whether the three electrically coupled lip motoneurons C15/16/17 are controlled by the buccal CPG or by a previously postulated cerebral CPG. Two buccal-cerebral projection interneurons, B34 and B63, which are part of the buccal CPG and mediate radula protraction, monosynaptically excite $\mathrm{C} 15 / 16 / 17$. Recordings from the $\mathrm{B} 34$ axon in the cerebral ganglion demonstrate its direct electrical coupling with C15/16/17, eliminating the need for a cerebral CPG. Moreover, when the multifunctional buccal CPG generates multiple forms of motor programs due to the activation of two inputs, the command-like neuron CBI-2 and the esophageal nerve (EN), $\mathrm{C} 15 / 16$ exhibit activity patterns that are distinct from C17. These distinct activity patterns result from combined activity of B34 and B63 and their differential excitation of $\mathrm{C} 15 / 16$ versus $\mathrm{C} 17$. In more general terms, we identified neuronal mechanisms that allow a single CPG to coordinate the phasing and activity of remotely located motoneurons innervating distinct structures that participate in the production of different motor outputs. We also demonstrated that axodendritic electrical coupling by projection interneurons plays a pivotal role in coordinating activity of these remotely located neurons.
\end{abstract}

\section{Introduction}

The graceful and orderly execution of complex movements often requires coordinated movements of multiple motor structures, which may be grouped roughly into two types. First, these structures may be similar to each other (e.g., body segments) and controlled by similar CNS oscillators or central pattern generators (CPGs), which are coordinated through coupling mechanisms (Williams, 1992; Kristan et al., 2005; Smarandache et al., 2009). Second, structures may be dissimilar, e.g., different limbs, or joints of a limb, or different feeding structures involved in grasping and/or digestion of food. Potentially, distinct motor structures may be controlled by different CNS regions, whose activity may be coordinated by one or multiple CPGs. Presently, there are suggestions that separate CPGs may be involved. For example, it was proposed that limbs and joints are controlled by separate CPGs (Grillner, 2006). Additionally, in well studied crustacean stomatogastric nervous systems (STNSs), separate CPGs control distinct feeding structures: e.g., the pylorus and gastric mill (Bartos et al., 1999). We study coordination between two feeding motor structures of the mollusk Aplysia: the outer

\footnotetext{
Received July 21, 2011; revised Sept. 4, 2011; accepted Sept. 9, 2011.

Author contributions: J.J. designed research; J.J., K.S., M.H.P., M.J.S., and B.C.L. performed research; B.C.L. contributed unpublished reagents/analytic tools; J.J. and K.S. analyzed data; J.J., E.C.C., and K.R.W. wrote the paper. This work was supported by NIH Grants NSO66587, NSO70583, and MH051393.

Correspondence should be addressed to Dr. Jian Jing, Department of Neuroscience, Box 1065, Mount Sinai School of Medicine, 1 Gustave Levy Place, New York, NY 10029. E-mail: jingj01@gmail.com.

DOI:10.1523/JNEUROSCI.3741-11.2011

Copyright $\odot 2011$ the authors $\quad 0270-6474 / 11 / 3115438-12 \$ 15.00 / 0$
}

lips that open and close, and the internal radula that protracts to grasp food while the lips are open. The Aplysia feeding network differs from the STNS in that lip-radula coordination occurs on a cycle-by-cycle basis, so there is one cycle period. Despite a previous suggestion to the contrary (Perrins and Weiss, 1996), we provide direct evidence that a single CPG may coordinate lip and radula movements. To our knowledge, this is a first description of a single CPG capable of coordinating movements of distinct motor structures.

Aplysia lip-radula coordination involves a complexity that lip and radula motoneurons are located in the separate cerebral and buccal ganglia, respectively. Consequently, the activity of these two sets of motoneurons has to be coordinated by projection neurons that have somata in one ganglion and axons projecting to the other. Radula motoneurons are controlled by a well characterized buccal CPG, but it is largely unknown how lip motoneurons are controlled. Perrins and Weiss (1996) suggested that lip-opening motoneurons are controlled by a cerebral CPG, based on their observation of cyclic activity in the cerebral ganglion disconnected from the buccal ganglion. They hypothesized that a central element of the cerebral CPG driving lip motoneurons is a cerebral-buccal interneuron (CBI), which had yet to be identified. However, the buccal CPG contains buccal-cerebral interneurons (BCIs) that mediate protraction (Hurwitz et al., 1997), and could coordinate the activity of cerebral lip motoneurons. This idea, in addition to the fact that neuronal axons can survive cutting of the somata in Aplysia (Evans et al., 2003), led us to examine an alternative hypothesis that a BCI axon accounts for 
the activity attributed to the putative cerebral CPG. Identifying the primary excitatory inputs to the lip motoneurons also allowed us to examine how various patterns of lip motoneuron activity during the generation of multiple forms of motor programs may be implemented by the BCIs. We discuss implications of our findings in the broader context of activity coordination between neurons located in different ganglia or brain regions.

\section{Materials and Methods}

Experiments were performed on Aplysia californica (100-250 g) obtained from Marinus. Aplysia are hermaphroditic; i.e., they have reproductive organs normally associated with both male and female sexes. Animals were anesthetized by injection of isotonic $\mathrm{MgCl}_{2}$ (30-50\% of body weight). The cerebral and buccal ganglia were removed, desheathed (ventral surface of the cerebral ganglion, caudal surface of the buccal ganglion), and pinned in a recording chamber $\left(14-17^{\circ} \mathrm{C}\right.$; volume, $\sim 1$ $\mathrm{ml}$ ) perfused at $0.33 \mathrm{ml} / \mathrm{min}$. The composition of normal artificial seawater was the following (in mM): $460 \mathrm{NaCl}, 10 \mathrm{KCl}, 11 \mathrm{CaCl}_{2}, 55 \mathrm{MgCl}_{2}$, and 10 HEPES, pH 7.6.

To suppress polysynaptic pathways, a high-divalent cation saline was used (in mM: $368 \mathrm{NaCl}, 8 \mathrm{KCl}, 13.8 \mathrm{CaCl}_{2}, 115 \mathrm{MgCl}_{2}$, and $10 \mathrm{HEPES}, \mathrm{pH}$ 7.6). In some experiments, ganglia were perfused with the high-divalent cation saline and then a $0 \mathrm{Ca}$ saline (in mM: $368 \mathrm{NaCl}, 8 \mathrm{KCl}, 128.8$ $\mathrm{MgCl}_{2}$, and 10 HEPES, pH 7.6) to block chemical synaptic transmission. The GABA antagonist $1(S), 9(R)$-( - )-bicuculline methobromide (a soluble form of bicuculline), the cholinergic blocker hexamethonium chloride, and other reagents were all obtained from Sigma. Drugs were dissolved into saline immediately before experiments.

Electrophysiology. Initially, intracellular recordings were made using single-barrel electrodes (4-10 M $\Omega$ ) filled with $2 \mathrm{M} \mathrm{K}$-acetate and $30 \mathrm{~mm}$ $\mathrm{KCl}$. Later experiments used electrodes filled with $0.6 \mathrm{M} \mathrm{K}_{2} \mathrm{SO}_{4}$ plus 60 mм KCl. Intracellular signals were acquired using an AxoClamp 2B amplifier (MDS Analytical Technologies) or a Getting model 5A amplifier (Getting Instruments). Extracellular signals were acquired from polyethylene suction electrodes using a differential alternating current amplifier (model 1700; A-M Systems). A Grass stimulator model S88 (Grass Instruments) was used for stimulation.

Neurons, including the cerebral lip motoneurons (Perrins and Weiss, 1996), were identified as described previously (Rosen et al., 1991; Church and Lloyd, 1994; Hurwitz et al., 1994, 1997; Jing and Weiss, 2001).

Intracellular recordings and photoablation of axons. To record from axons of B34, we used electrodes with a higher resistance (10-20 M $\Omega$ ). To facilitate localization of B34 axons, and also to avoid the much larger MCC axon located just anterior to the B34 axon, we injected carboxyfluorescein dye (instead of Alexa 488, because carboxyfluorescein diffuses faster) into the MCC. We visualized its axon in an electrophysiological setup using a yellow filter (model X-pro X001, Cokin) on the eyepiece of a stereomicroscope. In some experiments, we also visualized the axon by illuminating the live preparations with Luxeon V LED $(470 \mathrm{~nm})$ through a fiber optic cable. During the experiments, the microelectrode was aimed just posterior to the MCC axon to impale B34 axon.

For photoablation of B34 axons, we injected carboxyfluorescein dye into the B34 soma, and allowed the dye to diffuse into the cerebral ganglion overnight. To prevent leakage of carboxyfluorescein, we added an anion pump inhibitor, probenecid, in the dye solution. The B34 axon was ablated in the recording chamber by shining bright light (EX: 450-490 $\mathrm{nm}$ ) under a fluorescence microscope (Nikon) for 10-20 min until the axon was no longer visible. The recording chamber was then moved back to the electrophysiological rig to examine the functional effects of photoablation.

Identification of the type of motor programs. Motor programs were elicited via intracellular stimulation of cerebral-buccal interneuron-2 (CBI-2) at $\sim 10 \mathrm{~Hz}$. Each current pulse was set to trigger a single action potential. CBI-2 stimulation was manually terminated after the protraction phase ended as determined by activity in the I 2 nerve. Motor programs were also elicited extracellularly via stimulation of the esophageal nerve $(\mathrm{EN})$ at $2 \mathrm{~Hz}, 3 \mathrm{~ms}, 8-12 \mathrm{~V}$.
The buccal CPG generates several types of motor programs. The major components of these programs are protraction-retraction and openingclosing movements of the radula. Regardless of the type of motor program, protraction-retraction occurs in a fixed sequence in which protraction precedes retraction. Protraction was monitored by recording from the I2 nerve, which contains the axons of protractor motoneurons B31/32 and B61/62 (Hurwitz et al., 1994). In some experiments, retraction was monitored by recording from the buccal nerve 2 , which contains the axons of retractor motoneurons. In other experiments, retraction was monitored by the sustained depolarization of the radula-closing motoneuron B8 following protraction. In contrast to the phase-invariance of the protraction-retraction sequence, the timing of radula openingclosing depends on the type of program (Morton and Chiel, 1993a,b; Church and Lloyd, 1994; Nargeot et al., 1997; Jing and Weiss, 2001; Morgan et al., 2002; Jing et al., 2010; Wu et al., 2010). Briefly, when B8 fires predominantly during retraction, and thus (in the intact animal) helps bring the food into the buccal cavity, the program is considered to be ingestive. When $\mathrm{B} 8$ fires predominantly during protraction, and thus helps in removing an inedible object from the buccal cavity, the program is considered to be egestive. When B8 fires during both protraction and retraction, programs are defined as intermediate.

Data analysis. Electrophysiological recordings were digitized online with Axoscope and plotted with CorelDraw. Bar graphs were plotted using Axum (Mathsoft). Data are expressed as means \pm SEMs. Statistical tests were performed using Prism (GraphPad Software). They included Student's $t$ test and repeated-measures one-way ANOVA, which were applied as was appropriate. Data that showed significant effects in ANOVA were further analyzed in individual comparisons with Bonferroni's correction. In all statistical tests, effects were considered statistically significant when $p<0.05$.

\section{Results}

\section{Movement coordination of the radula and lips}

One central issue in the motor control of feeding in Aplysia is the coordination of lip and radula movements. The lips are the external feeding structure that can be opened or closed, while the radula is an internal feeding apparatus inside the buccal mass that moves forward (protracts) or backward (retracts) to manipulate food objects. Consequently, in order for the radula to have access to food that is outside the mouth, the lips need to be open as the radula protracts.

Lip-opening motoneurons (C15/16/17) are located in the cerebral ganglion (Perrins and Weiss, 1996). Radula movements are induced by the activity of motoneurons that are located in the buccal ganglion (Church and Lloyd, 1994; Hurwitz et al., 1994). The cerebral and buccal ganglia are connected by cerebral-buccal connectives (CBCs) (Fig. $1 B, C$ ). The activity of radula motoneurons, including neurons that mediate radula protraction, are controlled by a well characterized CPG in the buccal ganglion, and most elements of the buccal CPG have been identified (Susswein and Byrne, 1988; Rosen et al., 1991; Hurwitz and Susswein, 1996; Hurwitz et al., 1997; Jing and Weiss, 2001, 2002; Jing et al., 2004; Sasaki et al., 2009). Until now, relatively little has been known about the control of lip motoneurons.

\section{Nature of a putative cerebral CPG}

Previous work (Perrins and Weiss, 1996) postulated the existence of a putative cerebral CPG that would provide excitatory input to the lip motoneurons. An important finding was that, in the absence of the buccal ganglion, DC stimulation of $\mathrm{C} 15$ can drive cyclic activity in other lip motoneurons $\mathrm{C} 16 / 17$, i.e., cerebral motor programs (CMPs) (Fig. 1A). However, the CMP can be elicited by other means when $\mathrm{C} 15$ is hyperpolarized (Perrins and Weiss, 1996), suggesting that it is not mediated by C15. Rather, the CMP was hypothesized to be mediated by a cerebral interneu- 

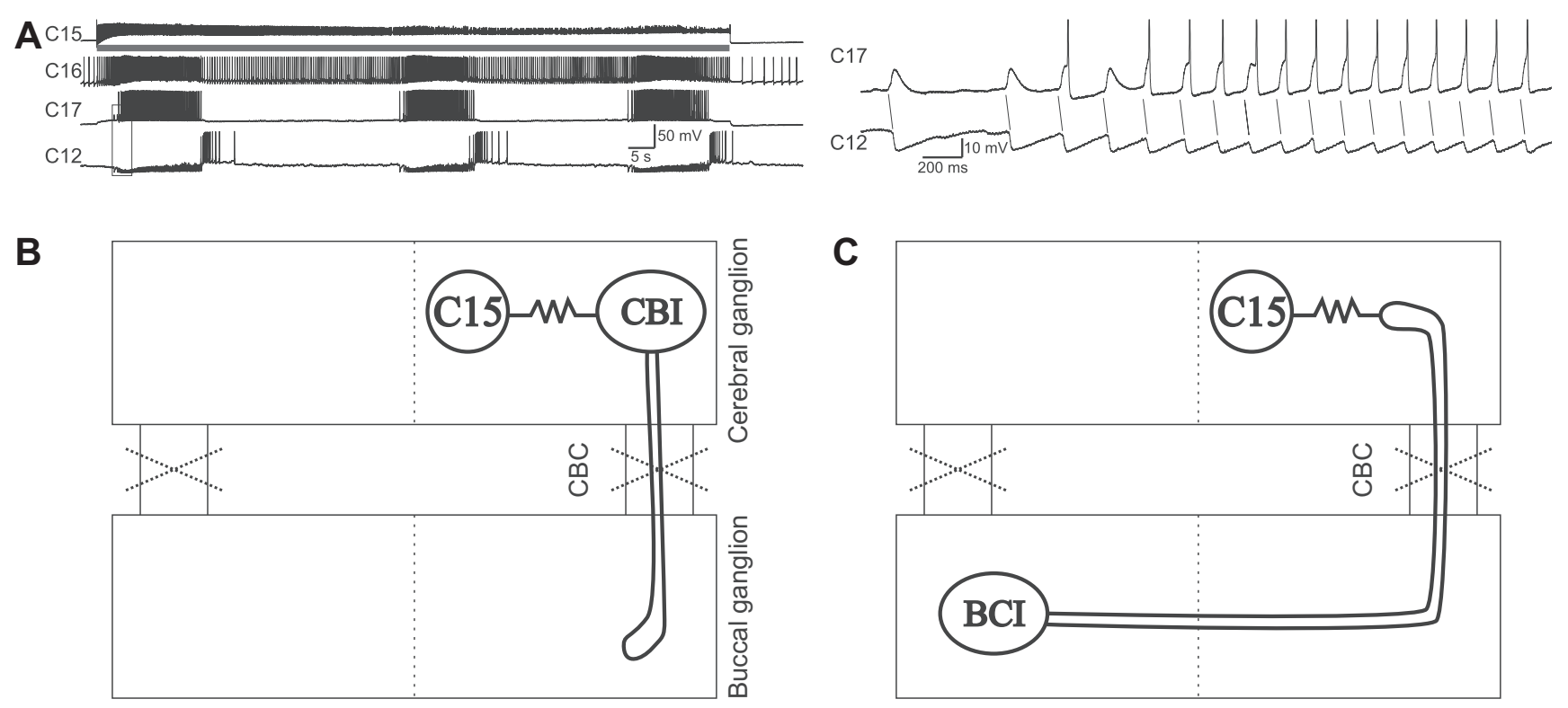

Figure 1. CMPs elicited by DC stimulation of $\mathrm{C} 15$ and simplified diagrams illustrating two alternative hypotheses accounting for the induction of CMPs. $A$, Left, DC stimulation of C15 (bar) elicited three cycles of bursting activity in C16 and C17, with each cycle corresponding to a barrage of fast IPSPs present in C12. Right, Enlarged records (indicated by the box at left) showing that the fast IPSPs in C12 corresponded one-for-one with EPSPs in C17. Note that the EPSPs in C17 preceded the IPSPs in C12. Spikes in C17 at right were clipped. B, The hypothesis postulated by Perrins and Weiss (1996): a CBI that is electrically coupled to C15 mediates the induction of CMPs in the isolated cerebral ganglion. C, The hypothesis that is being tested in this paper: the surviving axon of a BCl that is electrically coupled to C15 mediates the induction of CMPs. All cells in $\boldsymbol{B}$ and $\boldsymbol{C}$ are bilateral, but, only the cells on one side are depicted for simplicity.

ron, an element of a putative cerebral CPG, that is electrically coupled to C15 (Fig. $1 B$ ). This suggestion stems from the idea that DC stimulation of $\mathrm{C} 15$ can activate the postulated cerebral interneuron. One convenient monitor of the activity of this putative cerebral interneuron is the barrage of large-amplitude IPSPs in the cerebral motoneuron $\mathrm{C} 12$ that correspond one-forone with EPSPs in C15/16/17 (Fig. 1 A, right). Perrins and Weiss (1996) suggested that the cerebral interneuron is a CBI (Fig. 1B) because they found that the large-amplitude IPSPs in C12 correspond one-for-one with spikes in the $\mathrm{CBC}$. Characterizing the nature of the putative cerebral CPG is important because if such a CBI exists, its excitatory drive to lip motoneurons may influence lip-radula coordination.

Despite significant efforts, a CBI has never been identified that elicits EPSPs in C15/16/17 and IPSPs in C12. Tested CBIs include CBI-1, $-2,-3$, and -4 (Perrins and Weiss, 1996), CBI-5/6 (Perrins and Weiss, 1998), and CBI-8/9, -10, -11, and -12 (our unpublished observations). We therefore sought to examine an alternative hypothesis: that activity of the putative cerebral CPG can be accounted for by the axon of a BCI, which has its soma in the buccal ganglion but sends its axon to the cerebral ganglion (Fig. $1 C$ ). Specifically, because it is now known that the axons of $A p$ lysia neurons can survive after being separated from their somata by mechanical means (Evans et al., 2003), we postulated that the axon of the BCI might survive the cutting of the $\mathrm{CBC}$. If so, $\mathrm{DC}$ stimulation of $\mathrm{C} 15$ might activate the $\mathrm{BCI}$ axon in the isolated cerebral ganglion.

More specifically, if our hypothesis were correct, the $\mathrm{BCI}(\mathrm{s})$ should meet the following criteria: (1) the $\mathrm{BCI}(\mathrm{s})$ should provide strong excitatory input to $\mathrm{C} 15 / 16 / 17$; (2) the excitatory connections to $\mathrm{C} 15 / 16 / 17$ should be at least partly electrical, allowing DC stimulation of $\mathrm{C} 15 / 16 / 17$ to activate the $\mathrm{BCI}(\mathrm{s})$; (3) the $\mathrm{BCI}(\mathrm{s})$ should elicit large-amplitude IPSPs in C12; (4) when a CMP is activated, we should be able to record antidromic spikes, from its soma, that correspond, one-for-one, with the largeamplitude IPSPs in $\mathrm{C} 12$. Moreover, the $\mathrm{BCI}(\mathrm{s})$ should be neces- sary for the activation of the CMP. The experiments described in the following sections deal with these issues and, for convenience, are numbered as are the above criteria.

\section{(1) Synaptic output of BCIs to C15/16/17}

We focused on BCIs that are active during radula protraction because the lips are open when the radula protracts. Four such buccal-cerebral projection interneurons, termed protractionphase BCIs, have been identified. These cells are as follows: B34, B63, B40, and B20 (Teyke et al., 1993; Hurwitz et al., 1997; Jing and Weiss, 2001, 2002). In particular, B34 and B63 provide strong excitatory inputs to protraction motoneurons, and either are necessary for the initiation of protraction (Hurwitz et al., 1997; Dembrow et al., 2004; Jing et al., 2007) or can influence parametric features of protraction (Jing et al., 2003).

Among the four protraction-phase BCIs listed above, B34, $\mathrm{B} 63$, and $\mathrm{B} 40$ were found to induce one-for-one EPSPs in lip motoneurons (Fig. 2A). Because B34, B63, and B40 send axons only to the contralateral cerebral ganglion, and their axons in the cerebral ganglion do not cross the midline, synaptic inputs to the cerebral lip motoneurons are strictly contralateral (Fig. 1C). B34 and B63 elicited the largest EPSPs in C15/16/17 $(n=6)$ and thus are the focus of the remainder of this paper.

\section{(2a) B34 and B63 synaptic connections with C15/16/17 are at least partly electrical}

B34 and B63 are cholinergic; i.e., hexamethonium blocks the fast EPSPs that these neurons elicit in their postsynaptic followers in the buccal ganglion (Hurwitz et al., 2003). We therefore attempted to use hexamethonium to block the EPSPs that B34 and $B 63$ elicit in C15/16/17. We found that hexamethonium was ineffective in blocking the EPSPs that B34 elicits in $\mathrm{C} 15(n=3)$ (Fig. $2 \mathrm{B1}$ ). These data suggest that the EPSPs from B34 are purely electrical. The fast EPSPs from B63 appeared to have two components, an early component and a later slower one (Fig. 2B2). Earlier work (Hurwitz et al., 1997) that studied connections between B63 and other neurons to which it is electrically coupled 

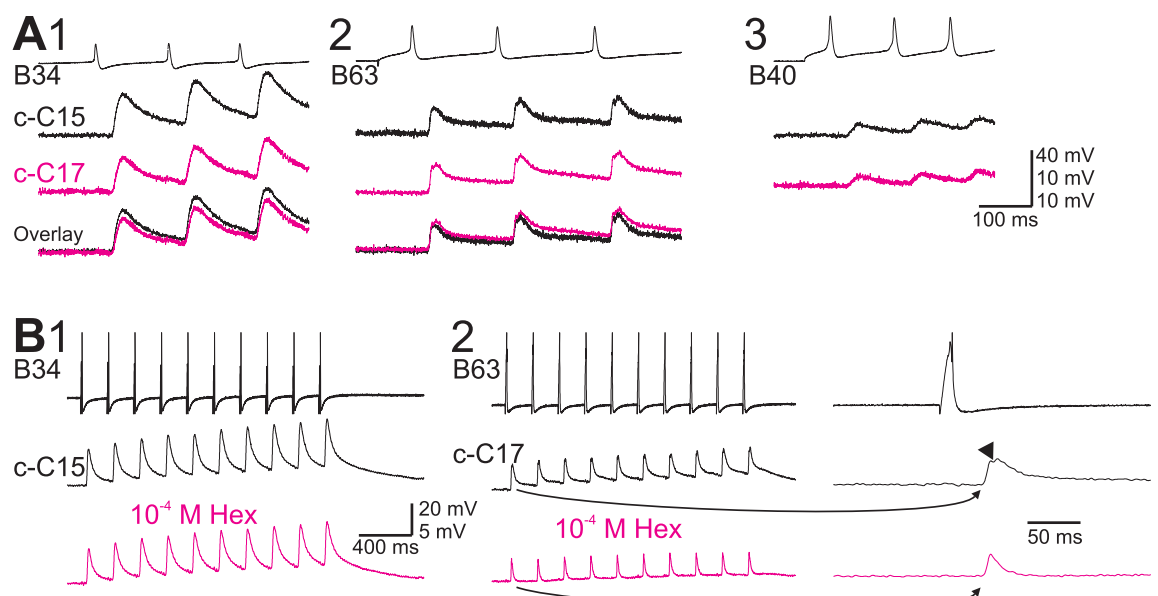

Overlay
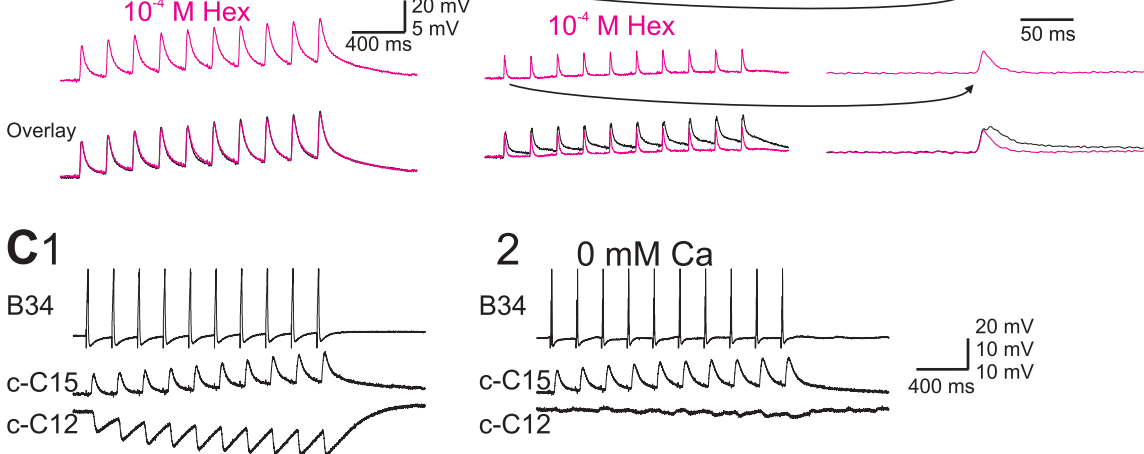

Figure 2. Synaptic connections from $B C l s(B 34, B 63)$ to lip motoneurons and indirect evidence suggesting that $B 34$ and $B 63$ connections to C15/16/17 are at least partly electrical. A1, B34 elicited monosynaptic EPSPs in the contralateral C15/17, with larger amplitude EPSPs in C15. A2, B63 elicited monosynaptic EPSPs in the contralateral C15/17, with larger amplitude EPSPs in C17. A3, B40 elicited small monosynaptic EPSPs in the contralateral C15/C17. Note that the rising phases of the EPSPs from B34 and B63 to C15/17 appeared to be faster than those of the EPSPs from B40 to C15/17. B1, The cholinergic blocker, $10^{-4} \mathrm{M}$ hexamethonium (Hex), had no effect on B34-elicited EPSPs in C15. B2, Hexamethonium had no effect on the early component of C17 EPSPs elicited by $B 63$, but did reduce the amplitude of the slower component (Expanded records at right: arrowhead), suggesting that the connection from B63 to C17 might be partly electrical. C, B34 elicited fast EPSPs in C15 and fast IPSPs in C12 (C1). Perfusion of 0 mm Ca had no obvious effect on EPSPs in C15, but reduced the amplitude of IPSPs in C12 (C2). Recordings in $\boldsymbol{A}$ were made in normal saline. All other recordings were made in high-divalent saline except the recordings in $\mathbf{C}$, which were made in $\mathbf{0}$ Ca saline. Throughout figures, "c" before cell names: contralateral.

(B31/32) had shown that this connection also has two components. The early component appears to be electrical, while the later component is chemical. We found that hexamethonium decreased the later component of B63 to C17 EPSPs without affecting the early component $(n=3)$ (Fig. 2B2). This is consistent with the possibility that the early component of the EPSP is electrical.

To seek further evidence that B34-elicited EPSPs in C15/16/17 are electrical, we tested the effect of 0 Ca saline (Fig. 2C), which blocks chemical synaptic transmission but has little effect on electrical transmission. We included $\mathrm{C} 12$ as a control because B34 elicits IPSPs in $\mathrm{C} 12$ that are chemical (e.g., are blocked by bicuculline, see Fig. 5C). As expected, we found that $0 \mathrm{Ca}$ saline reduced the amplitude of these IPSPs (Fig. 2C2). In contrast, it had little effect on B34-elicited EPSPs in C15/16/17. The above data provide indirect support for the hypothesis that B34 and B63 are electrically coupled to lip motoneurons.

\section{(2b) Direct electrical coupling between}

the B34 axon in the cerebral ganglion and C15/16/17

Initially, we attempted to obtain direct evidence of electrical coupling between B34 and lip motoneurons by hyperpolarizing the soma of B34. We did not, however, observe appreciable changes in the membrane potential of C15/16/17 (or vice versa). Because the B34 soma is far away from its cerebral axon (and therefore the soma and the cerebral axon may be electrotonically separated), these data cannot be used as evidence against electrical coupling. Thus, we sought to obtain direct evidence for electrical coupling by recording from the $\mathrm{B} 34$ axon in the cerebral ganglion, which is close to the processes of C15/16/17

Toward this end, we localized the B34 axon in the cerebral ganglion by injecting a fluorescent dye into the B34 soma. We observed that the B34 axon was near the ventral surface of the cerebral ganglion, was relatively superficial and large, and was located toward the posterior part of the root of the CBC where it entered the cerebral ganglion (Fig. 3B2). This is a region that previous data suggested may also contain the axon of the MCC, a giant cell in the cerebral ganglion (Weiss and Kupfermann, 1976). Indeed, when we performed double labeling experiments by injecting fluorescent dyes in both the MCC (Alexa 488, green) (Fig. 3A1,B1) and B34 (Alexa 568, red) (Fig. 3B2), we found that the MCC axon was much larger than the B34 axon and was just anterior to it (Fig. 3B3). The close proximity of the two axons facilitated the impalement of the B34 axon. Specifically, in live preparations, we injected carboxyfluorescein dye into the MCC, and were able to visualize the MCC axons (within $2 \mathrm{~h}$ ) (Fig. $3 B 4$ ). We then attempted to impale axons located just posterior to the MCC axon. An example of a successful recording of the B34 axon using this method is shown in Figure $4(n=3)$. By hyperpolarizing the B34 axon, we observed large negative deflections in the membrane potential of $\mathrm{C} 15$, and vice versa (Fig. $4 C)$. There was an almost complete absence of voltage change in B34 soma. The coupling ratio from the B34 axon to C15/16/17 $(0.44 \pm 0.03)$ is larger than from $\mathrm{C} 15 / 16 / 17$ to $\mathrm{B} 34$ axon $(0.22 \pm$ $0.01, n=3$ ). Because C15/16/17 are all electrically coupled (Perrins and Weiss, 1996), we conclude that the B34 axon is electrically coupled to all three lip motoneurons. It is notable that there was no obvious afterhyperpolarization in the B34 axonal spikes (Fig. 4A). This may be one reason that the electrical connections from B34 to C15/16/17 appear to be primarily excitatory (Fig. 2), because afterhyperpolarization in presynaptic neurons may induce an inhibition in electrically coupled postsynaptic cells (Galarreta and Hestrin, 2001).

\section{(3) Synaptic output of B34 and B63 to C12}

Both B34 (Fig. 5A) and B63 (Fig. 5B) elicited IPSPs in C12 that followed presynaptic spikes one-for-one. Typically, the $\mathrm{C} 12$ IPSPs elicited by B34 underwent facilitation when B34 was stimulated at $5-10 \mathrm{~Hz}$. The amplitude of unfacilitated IPSPs $(n=7)$ was as follows: $-3.14 \pm 0.73 \mathrm{mV}$, facilitated IPSPs $(n=4)$ : $-7.15 \pm 1.57 \mathrm{mV}$. In contrast, the C12 IPSPs elicited by B63 underwent little facilitation with an amplitude of $-1.04 \pm 0.16$ $\mathrm{mV}(n=5)$. Notably, the C12 IPSPs elicited by B63 were smaller than even the unfacilitated IPSPs elicited by B34 (unpaired $t$ test, $p<0.05$ ). Interestingly, the facilitated C12 IPSPs elicited by B34 
$(-7.15 \pm 1.57 \mathrm{mV})$ were somewhat smaller but comparable to the C12 IPSPs when CMPs were induced by DC stimulation of C15/16/17 (Fig. $1 A,-7.75 \pm 0.55 \mathrm{mV}, n=11$, unpaired $t$ test, $p>0.05)$. These data support the notion that B34 and B63 may function as critical elements of the putative cerebral CPG. Because the C12 IPSPs elicited by B34 are more comparable in amplitude to the IPSPs present in CMPs, B34 may be more important than B63.

Previous work has shown that B34 is both GABAergic (Jing et al., 2003) and cholinergic (Hurwitz et al., 2003) in that it uses GABA to elicit IPSPs in the buccal retraction interneuron B64, and ACh to elicit fast EPSPs in buccal protraction neurons B31/ 32. We therefore examined the possibility that the C12 IPSPs elicited by B34 might be GABAergic. We applied the $\mathrm{GABA}_{\mathrm{A}}$ receptor antagonist, $1 \mathrm{~mm}$ bicuculline, and found that it decreased the amplitude of the IPSPs in $\mathrm{C} 12$ (Fig. $5 C, n=3$ ). These data suggest that B34 may use GABA to mediate inhibitory transmission to $\mathrm{C} 12$.

(4) Role of B34 in the activation of CMPs

To summarize, both B34 and B63 make the synaptic connections that were predicted for the postulated element of the cerebral CPG, but only B34-induced large-amplitude IPSPs are comparable in amplitude to the IPSPs observed during C15-evoked CMPs. This suggests that B34 participates in CMPs. To test this idea, we first determined whether B34 was sufficient to elicit CMP-like activity. We found that when the B34 soma was depolarized via DC current injection, we evoked cyclic activity in both the $\mathrm{B} 34$ axon and $\mathrm{C} 15$ (Fig. 6) resembling the previously recorded CMPs (Fig. 1A). This indicates that B34 activity is sufficient for CMP induction.

We then performed experiments in which we drove one cycle of a CMP (as evidenced by a barrage of large-amplitude fast IPSPs in C12) through DC depolarization of C15/16/17, while recording from the soma of the contralateral B34 (Fig. 7A). We found that each IPSP in C12 corresponded one-for-one with antidromic spikes in B34 $(n=8)$. Furthermore, the onset of the IPSP preceded the onset of the antidromic spike in B34 soma as expected since B34 spikes were initiated in the cerebral ganglion (Fig. 7A, right). Conversely, when the $\mathrm{B} 34$ soma was stimulated and B34 spikes were initiated in the buccal ganglion, IPSPs in C12 and EPSPs in C15/16 occurred after the B34 somatic spikes (Fig. $7 B$, right). B34 antidromic spikes during the activation of CMPs (Fig. $7 A$ ) and B34-elicited IPSPs in C12 (Figs. $5 A, 7 B$ ) would also account for the spikes in the $\mathrm{CBC}$ that correspond one-for-one with the IPSPs in C12 when CMPs are activated by DC stimulation of C15 (Perrins and Weiss, 1996) (see also Fig. 1C).

When we recorded from somata of the contralateral B34 and B63, and activated CMPs through DC stimulation of C15 $(n=3)$, we always observed antidromic spikes in the B34 soma, but never observed any antidromic spikes in the B63 soma (data not shown). This is further consistent with the idea that only B34 is activated during CMPs.

The above data support the hypothesis that the B34 axon can account for the activity of the hypothesized critical element of the cerebral CPG (Perrins and Weiss, 1996). To provide further evidence that this is the case, we examined whether B34 is necessary
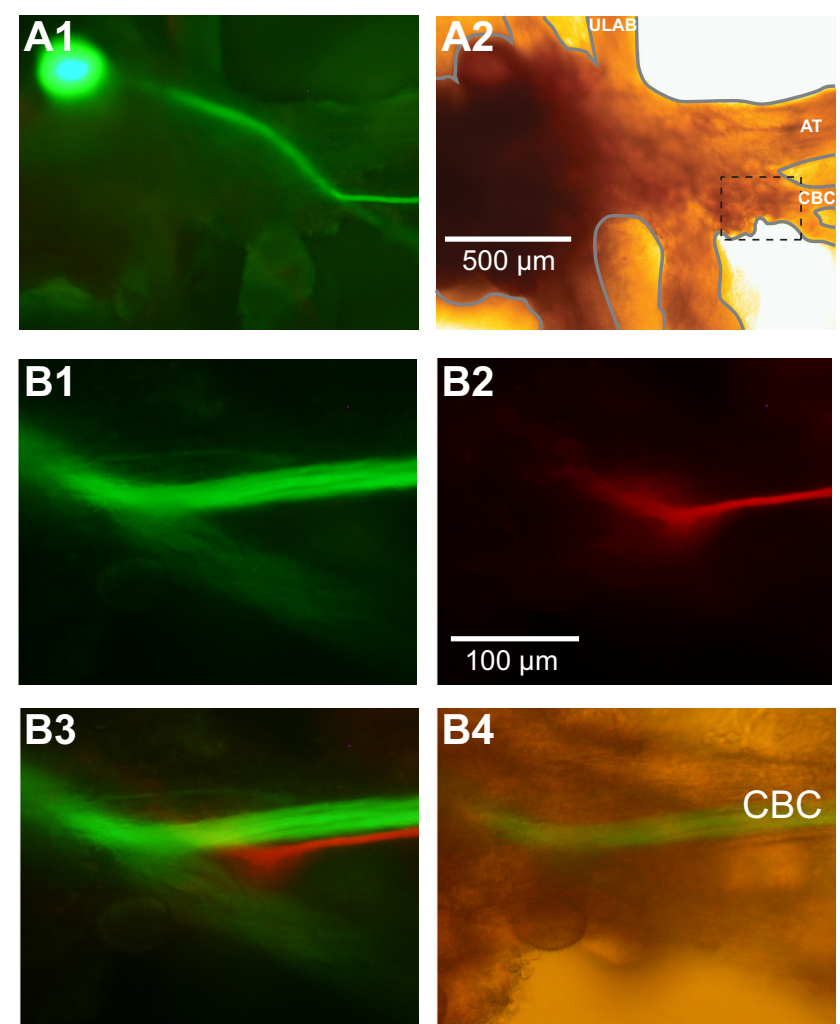

Figure 3. Localization of the $B 34$ axon in the cerebral ganglion and its position relative to MCC axon. A, Right cerebral ganglion at lower magnification. A1, Dye (Alexa 488) injection in the right MCC showing its soma and axonal projections; $\boldsymbol{A 2}$, Same view as $\boldsymbol{A 1}$, except photographed with trans-illumination. $\boldsymbol{B}$, The $B 34$ axon near the root of the $C B C$ in the cerebral ganglion at higher magnification (indicated by the box in $\mathbf{A 2}$ ). B1, The MCC axon (green); B2, The B34 axon (red). The B34 soma in the left buccal ganglion was injected with Alexa 568, which diffused into the $B 34$ axon in the $C B C$ and the right cerebral ganglion; $\boldsymbol{B} 3$, overlay of $\boldsymbol{B} 1$ and $\boldsymbol{B} 2$; $\boldsymbol{B} 4$, the same as $\boldsymbol{B} \mathbf{1}$, except that the trans-illumination was also turned on. This view illustrates electrophysiological experiments when only the MCC was injected with carboxyfluorescein, and a microelectrode was aimed just below the MCC axon to impale the B34 axon. AT, Anterior tentacular nerve; ULAB, upper labile nerve.

Figure 4. The $B 34$ axon in the cerebral ganglion is electrically coupled to lip motoneurons. $A, D C$ stimulation of the $B 34$ soma induced orthodromic spikes in the B34 axon that followed B34 somatic spikes. Note B34 axonal spikes had little afterhyperpolarization. $\boldsymbol{B}$, DC stimulation of the B34 axon induced antidromic spikes in the B34 soma that followed B34 axonal spikes. $\boldsymbol{C}$, Hyperpolarization of the B34 axon (left) or C15 (middle) induced a corresponding hyperpolarization in the other neuron, but had no obvious effect on the membrane potential of the B34 soma, indicating that the B34 axon was electrically coupled to C15. Hyperpolarization of the B34 soma (right) had no obvious effect on the B34 axon or C15. Bars, The cells that were depolarized $(\boldsymbol{A}, \boldsymbol{B})$ or hyperpolarized $(\boldsymbol{C})$. The coupling ratio was larger from the B34 axon to C15 than from C15 to the B34 axon.

for C15-evoked CMPs by photo-ablating the B34 axon. For this experiment, we injected one B34 soma with carboxyfluorescein dye and incubated overnight so that the B34 axon in the cerebral ganglion was labeled (Fig. 8A2). After photoablation of the B34 axon (Fig. 8A3), the ipsilateral C15 was no longer able to drive a CMP, as evidenced by the absence of large-amplitude IPSPs in C12 (Fig. 8 B). In contrast, the contralateral C15 was still able to 

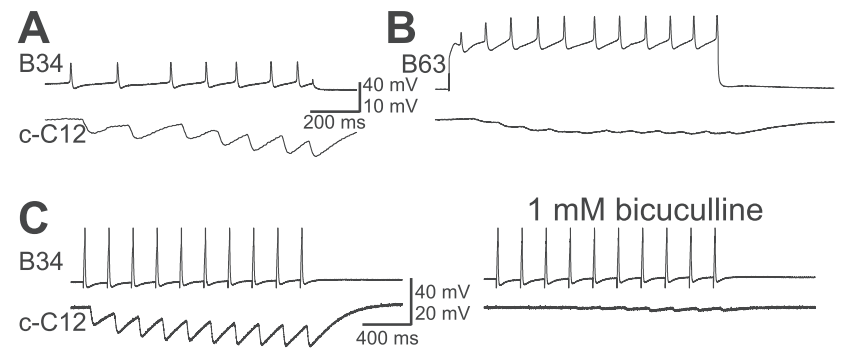

Figure 5. Synaptic connections from $B C l s$ ( $B 34$ and $B 63)$ to $C 12 . A, B$, Both $B 34(A)$ and $B 63$ $(B)$ elicited IPSPs in the contralateral C12. B34-induced IPSPs were, however, larger. C, B34 elicited one-for-one IPSPs in C12 (left), and perfusion of $1 \mathrm{~mm}$ bicuculline (right) decreased B34 to C12 IPSPs, suggesting that the connection may be mediated by GABA. Recordings in C were made in high-divalent saline.

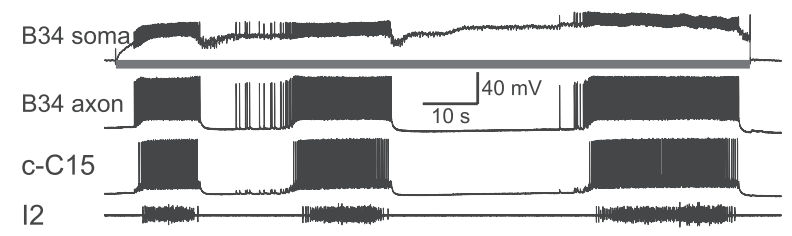

Figure 6. DC stimulation of the B34 soma can induce activity resembling the CMPs. Sustained depolarization of B34 soma (bar) induced cyclic activity (three cycles) in C15.

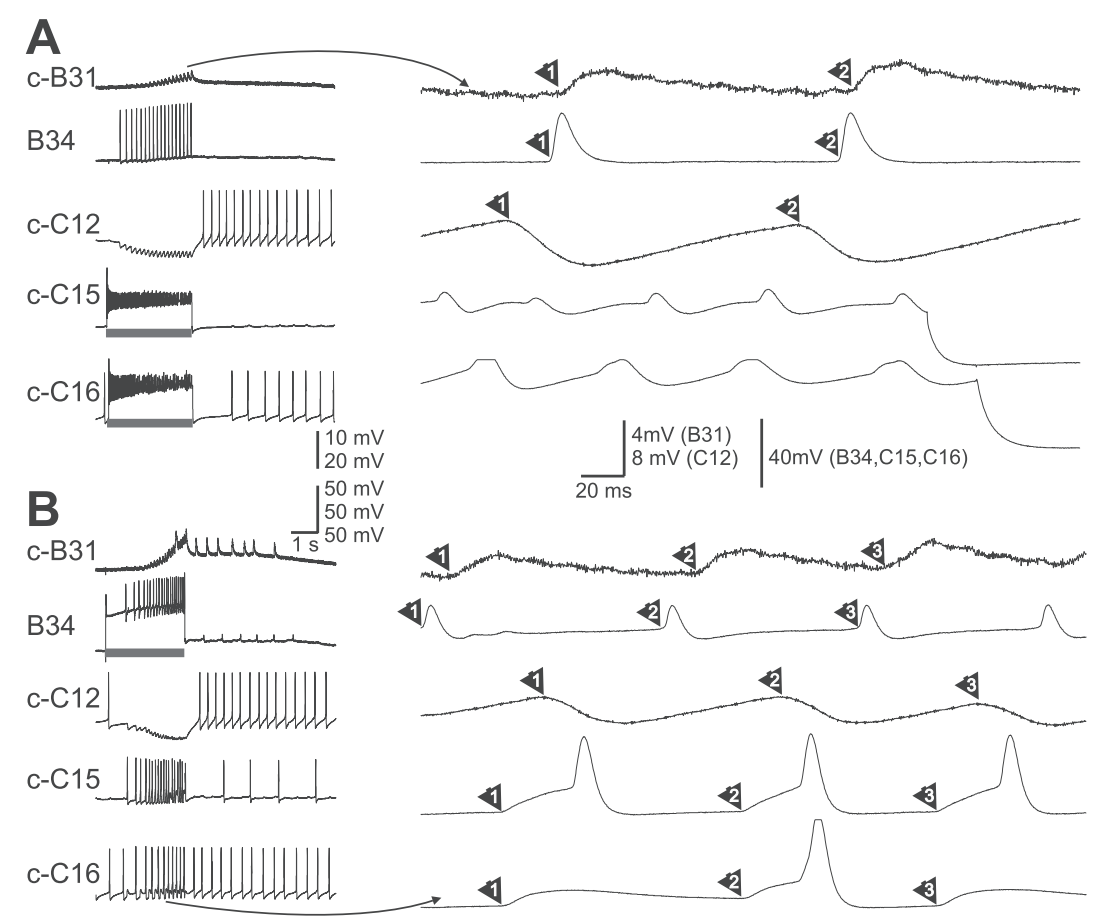

Figure 7. Induction of CMPs is associated with antidromic spikes in B34. A, DC stimulation of C15 and C16 (bars) induced one cycle of a CMP, which was associated with a barrage of large-amplitude IPSPs in C12 (left). Each IPSP in C12 (right) corresponded to and preceded an antidromic spike in the contralateral B34. In turn, each spike in B34 corresponded to and preceded an EPSP in buccal neuron B31, an excitatory postsynaptic follower of B34. B, DC stimulation of B34 (bar) induced EPSPs in B31, activated C15/16, and induced IPSPs in C12 (left). However, there were some differences in the timing of the PSPs compared with $\boldsymbol{A}$. Each spike in B34 corresponded to and preceded an EPSP in B31, that was followed by an EPSP in C15/16, and finally an IPSP in C12 (right).

drive a CMP $(n=3)$. Thus, the B34 axon is both necessary and sufficient for the activation of a CMP.

In summary, we demonstrate that the BCI (B34) satisfies the four Perrins/Weiss criteria for the missing cerebral CPG element; i.e., it makes excitatory connections with $\mathrm{C} 15 / 16 / 17$, the excit- atory connections are electrical, it makes an inhibitory connection with $\mathrm{C} 12$, and it is activated during CMPs. In effect, our data indicate that the cerebral CPG as postulated by Perrins and Weiss (1996) does not exist.

\section{Interneuronal basis of the activation of $\mathrm{C} 15 / 16 / 17$ during feeding motor programs}

A further prediction of our model is that when buccal motor programs (BMPs) are triggered, rhythmic activity in lip motoneurons will occur during protraction. (BMP induction will activate protraction-phase $\mathrm{BCIs}$, which will excite lip-opening motoneurons and inhibit C12.) In the isolated CNS, two major types of BMPs can be elicited: ingestive and egestive. Ingestive motor programs are elicited by stimulation of a command-like neuron, CBI-2, that is normally activated by food (Jing and Weiss, 2005). Egestive motor programs are elicited by stimulation of the EN, which innervates the esophagus and the gut (Chiel et al., 1986; Church and Lloyd, 1994). As expected, lip motoneurons are active during radula protraction during both ingestive and egestive programs (Fig. 9).

Earlier data (Perrins and Weiss, 1996) suggested that the activity of lip motoneuron $\mathrm{C} 15$ differs during ingestive and egestive programs (Fig. 9). We recorded the activity of all three lip motoneurons (C15/16/17) during CBI-2 and EN motor programs and found that $\mathrm{C} 15$ and $\mathrm{C} 16$ showed identical patterns of firing, while $\mathrm{C} 17$ differed. Specifically, C15/16 fired most strongly during CBI-2-elicited ingestive programs $(8.59 \pm 0.74 \mathrm{~Hz}, n=5)$ but was only weakly active during EN-elicited egestive programs $(2.92 \pm 0.73 \mathrm{~Hz}, n=$ 5). (Because of the similarity in $\mathrm{C} 15$ and C16 activity, we pooled data from C15 and C16.) In contrast, C17 fired strongly during CBI-2-elicited programs $(9.45 \pm 0.86 \mathrm{~Hz}, n=4)$, but was also very active during EN-elicited programs $(6.85 \pm 0.3 \mathrm{~Hz}, n=4)$.

The distinct firing patterns of $\mathrm{C} 15 / 16$ versus $\mathrm{C} 17$ in EN-elicited egestive motor programs suggested that BCIs might excite these lip motoneurons differentially. To determine whether this is the case, first, we examined the strength of synaptic inputs from $\mathrm{B} 34$ and $\mathrm{B} 63$ to $\mathrm{C} 15 / 16$ versus C17. For the synaptic inputs to be comparable, postsynaptic cells were maintained at the same membrane potential. We found that EPSPs from B34 to C15/16 $(8.02 \pm 1.46 \mathrm{mV})$ were larger than those from B34 to $\mathrm{C} 17$ ( $6.09 \pm 0.83 \mathrm{mV}, n=5$, paired $t$ test, $p<0.05$ ) (Fig. $2 A 1$ ), while EPSPs from B63 to C15/16 (5.18 \pm 0.53 $\mathrm{mV}$ ) were smaller than those from B63 to C17 (7.69 $\pm 1.09 \mathrm{mV}, n=7$, paired $t$ test, $p<0.05$ ) (Fig. 2A2). Thus, B34 appears to preferentially act on $\mathrm{C} 15 / 16$, while $\mathrm{B} 63$ appears to preferentially act on C17.

Second, we examined the contributions of B34 and B63 to the firing of the lip motoneurons, $\mathrm{C} 15 / 16$ or $\mathrm{C} 17$, during programs elicited by CBI-2. We elicited ingestive programs by stimulating CBI-2, and determined effects of hyperpolarization of either B34 or B63 on the activity of C15/16/17. B34 hyperpolarization had 
the most dramatic effect on the activity of C15/16, i.e., a $64 \%$ reduction $(8.09 \pm 0.39$ $\mathrm{Hz}$, vs $2.9 \pm 0.28 \mathrm{~Hz}, n=4$, paired $t$ test, $p<0.0001$ ) (Fig. 10A). C17 activity was reduced by $47.8 \%(10.54 \pm 0.47 \mathrm{~Hz}$, vs $5.5 \pm 0.36 \mathrm{~Hz}, n=5$, paired $t$ test, $p<$ 0.001). B63 hyperpolarization also had a significant, but less dramatic, effect on the activity of $\mathrm{C} 15 / 16$, i.e., a $12.2 \%$ reduction $(8.53 \pm 0.66 \mathrm{~Hz}$, vs $7.49 \pm 0.67 \mathrm{~Hz}, n=5$, paired $t$ test, $p<0.0001$ ) (Fig. $10 B$ ). C17 activity was reduced by $13.1 \%(8.93 \pm$ $0.82 \mathrm{~Hz}$, vs $7.76 \pm 1.03 \mathrm{~Hz}, n=4$, paired $t$ test, $p<0.05)$. These data indicate that both B34 and B63 contribute to the activation of $\mathrm{C} 15 / 16 / 17$ during CBI-2-elicited programs, but that $\mathrm{B} 34$ predominates.

Third, we attempted to determine the contribution of B63 to substantial activity of $\mathrm{C} 17$ during EN-elicited programs. However, B63 hyperpolarization prevented expression of complete EN programs, as evidenced by the lack of significant activity in $\mathrm{I} 2$ nerves and B8 (data not shown). Nonetheless, B63 may provide the primary excitatory input to C17 because B34 does not fire significantly during EN-elicited programs (see Fig. 12A2), whereas B63 shows highfrequency firing (see Fig. 12C2).

\section{Input versus state-dependent activity of lip motoneurons}

Ingestive and egestive programs are typically elicited by two different inputs: CBI- 2 and the EN respectively. Thus, the difference in activity of C15/16 in these two types of programs could result from either the driving input: i.e., $\mathrm{CBI}-2$ versus $\mathrm{EN}$, or the state, i.e., the type of program. To distinguish between these two possibilities, we used a biasing paradigm (Proekt et al., 2004, 2007; Friedman et al., 2009) that creates a situation in which CBI-2 elicits egestive, rather than ingestive programs. This is achieved by evoking a CBI-2-induced cycle of activity following a series of EN-elicited egestive programs.

In our biasing experiments, we first elicited eight cycles of motor programs by stimulating CBI-2. Motor programs became strongly ingestive, i.e., B8 fired strongly during retraction (Fig. 11 A1). The EN was then stimulated for 5 min to elicit $8-10$ cycles of egestive programs, the last of which is shown in Figure $11 \mathrm{~A} 2$. This cycle was egestive; i.e., B8 showed strong activity during protraction. Similar to our observations described in the last section, C15 showed weak activity while C17 showed substantial activity in EN-elicited programs. Subsequently, the input was switched back to CBI-2 and the program elicited by CBI-2 remained egestive (Fig. 11A3); i.e., B8 only fired during protraction. (If no stimulation were given during the $5 \mathrm{~min}$ period, CBI-2 would have elicited an ingestive motor program (Friedman et al., 2009).) Interestingly, in this program, both C15 and C17 showed activity levels comparable to that before EN stimu-
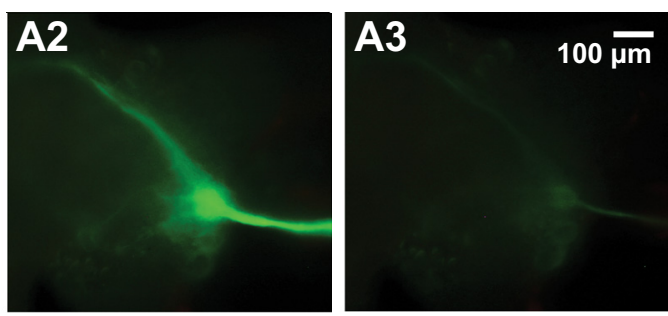

20 min after

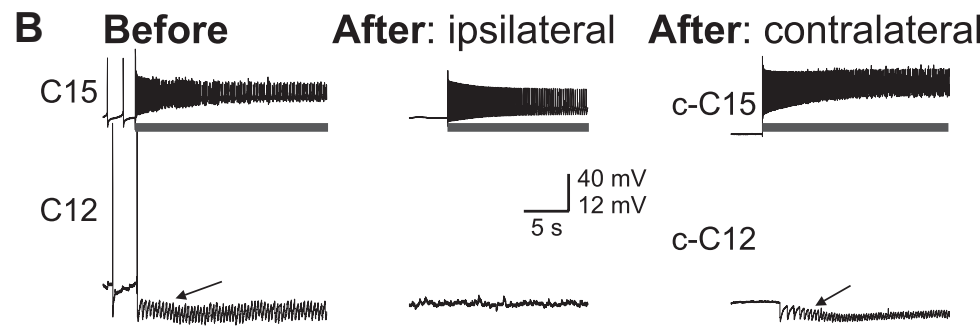

Figure 8. Effects of a lesion of the B34 axon on CMPs. A, Photoablation of the B34 axon. All photos are of the same region of the ight cerebral ganglion. A1, Bright-field image showing the general location of neuronal somata (C12, $\mathrm{C} 15)$ and nerves/connec-
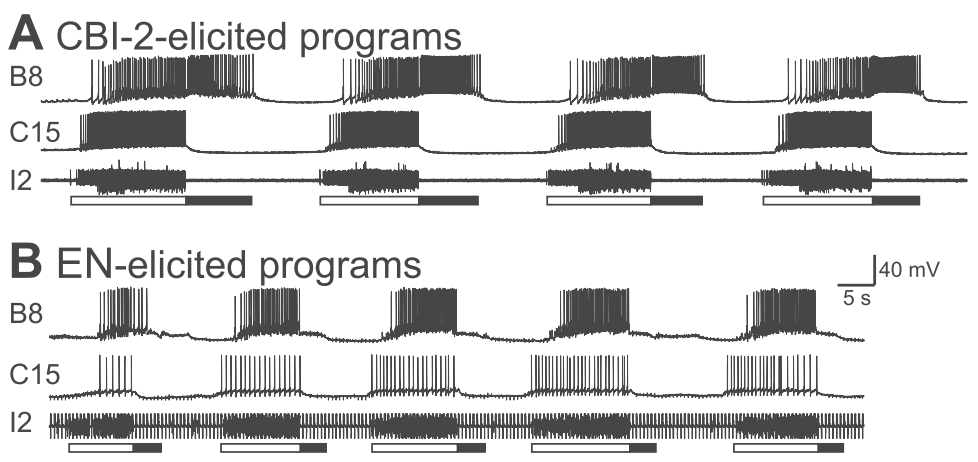

Figure 9. Activity of the lip motoneuron 15 during buccal motor programs. A, Multiple cycles of motor programs were elicited by stimulation of $\mathrm{CBI}-2$ (CBI-2 recording not shown). Programs were initially intermediate, i.e., B8 fired during both protraction (open bar) and retraction (filled bar), and became ingestive in later cycles, i.e., B8 fired predominantly during retraction. $\boldsymbol{B}$ (T) first program was intermediate, i.e., B8 fired during both protraction (open bar) and retraction (filled bar), and became egestive in later cycles, i.e., B8 fired only during protraction. In both $\boldsymbol{A}$ and $\boldsymbol{B}, \mathrm{C} 15$ fired during protraction, although it was more active during CBI-2-elicited programs than during EN-elicited programs.

lation. After eight more cycles of motor programs were produced by stimulating CBI-2, the firing frequency of all neurons returned to pre-EN level. Group data (Fig. $11 B$ ) indicate that activity of $\mathrm{C} 15 / 16\left(n=5, F_{(2,8)}=71.6, p<0.0001\right)$ and $\mathrm{C} 17\left(n=4, F_{(2,6)}=\right.$ $11.56, p<0.01$ ) was lower during EN programs, but their activity did not differ in CBI-2 programs elicited before versus after EN programs. These data suggest that $\mathrm{C} 15 / 16$ activity may be input specific (CBI-2), while C17 may be a shared element (in programs elicited by either CBI-2 or the EN) in that it is substantially activated independent of network input or the nature of the motor program.

In a final set of experiments, we examined the activity patterns of B34 versus B63 during the biasing paradigm. As described before (Jing and Weiss, 2001), B34 fired at high frequency during programs elicited by CBI-2 (Fig. 12A1). In addition, B34 was 


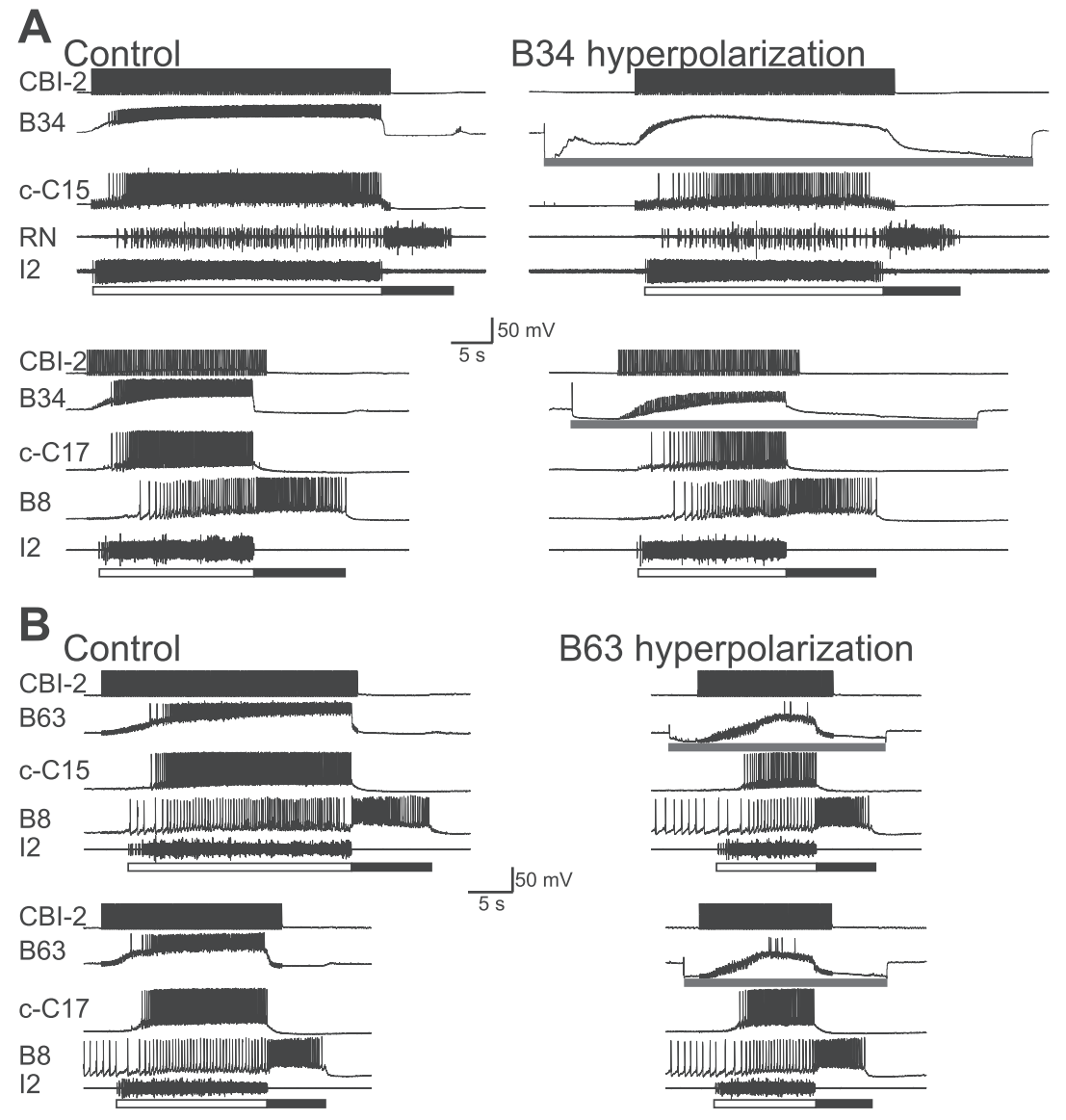

Figure 10. Contribution of $\mathrm{B} 34$ or $\mathrm{B} 63$ to the activation of $\mathrm{C} 15 / 16$ or $\mathrm{C} 17$ during $\mathrm{CBI}-2$-elicited motor programs. $A, \mathrm{~B} 34$ hyperpolarization (bars, right) reduced $\mathrm{C} 15$ (top) and C17 (bottom) activity during CBI-2-elicited programs. RN, Radula nerve; large units represent B8 activity. B, B63 hyperpolarization (bars, right) reduced C15 (top) and C17 (bottom) activity during CBI-2-elicited programs. B34 effects were stronger than B63 effects.

weakly or not active during egestive programs elicited by EN (Fig. 12A2). We did, however, find that it is strongly active in biased programs (egestive) elicited by CBI-2 after EN stimulation (Fig. 12A3) (Group data, Fig. $12 B, n=5, F_{(2,8)}=69.59, p<0.0001$ ). This activity pattern of B34 matches that of C15 and C16. In contrast, $\mathrm{B} 63$ is strongly active during programs elicited by both CBI-2 and the EN (Fig. 12C) (Group data, Fig. $12 D, n=5, F_{(2,8)}$ $=5.09, p<0.05)$. Although B63 showed somewhat higher firing during EN-elicited programs, Bonferroni's multiplecomparison test showed that the difference was not statistically significant. Because of strong firing of B63 during EN programs, it may contribute to the substantial firing of $\mathrm{C} 17$ during EN programs. To summarize, in biasing experiments, we demonstrate similarity between B34 and C15/16 activity, and $\mathrm{B} 63$ and $\mathrm{C} 17$ activity. This provides further support for the idea that B34 and B63 are critical drivers of C15/16/17 activity during feeding motor programs.

Together, our data indicate that distinct synaptic outputs and firing patterns during motor programs of two buccal CPG interneurons, B34 and B63, are the bases of the distinct firing patterns of lip motoneurons during feeding motor programs.

\section{Discussion}

Coordination of distinct motor structures: single or separate CPGs?

Coordination between multiple motor structures is essential for normal motor activity. Substantial efforts have been made to characterize mechanisms that coordinate movements of similar body parts, e.g., different body segments in model systems such as lamprey and leech swimming, crayfish swimmerets movements (see Introduction), and limb coordination in quadrupeds (Grillner, 1981) and insects (Ludwar et al., 2005). In segmental animals, an emerging consensus is that each segment is controlled by its own CPG, and coordination is implemented by coupling between these CPGs.

Coordination between dissimilar motor structures is of critical importance as illustrated by diverse examples that include the following: coordination between various feeding structures in Aplysia, crustaceans (Harris-Warrick et al., 1992), and frog (Nishikawa, 1999); human arm-leg coordination during locomotion (Dietz, 2002; Zehr and Duysens, 2004); and coordination of respiration and locomotion (Gariépy et al., 2010). However, the level of detail in these studies varies, perhaps reflecting significant variability in how tightly these different sets of movements are coupled. In some cases, separate CPGs may be involved. This is especially clear in the STNS, where separate CPGs control the pylorus and gastric mill. In vertebrates, the control of different limbs or joints of a limb is also proposed to involve separate CPGs (Grillner, 2006), although a clear demonstration of such a mechanism is not yet available (Dietz and Michel, 2009).

We investigated whether the control of two distinct feeding motor structures, the radula and lips, is implemented by a single CPG or two CPGs. Radula motoneuron activity is controlled by a buccal CPG. Perrins and Weiss (1996) hypothesized that activity of cerebral lip motoneurons is controlled by a putative cerebral CPG based on the ability of C15/16/17 to drive CMPs in the absence of the buccal ganglion (Fig. 13A). In Pleurobranchaea, the existence of a cerebral oscillator has also been suggested (Davis et al., 1984). Here, we found that Aplysia protraction-phase BCIs (B34, B63) drive C15/16/17. Moreover, recordings of the B34 cerebral axon indicate that it is electrically coupled to $C 15 / 16 / 17$ (Fig. 13B). Thus, CMPs can be explained by the survival of this axon after cutting the CBCs. We also demonstrate an inhibitory connection from B34 to $\mathrm{C} 12$ that was observed for the proposed cerebral CPG element. Finally, we demonstrate an obligatory role of the B34 axon in CMPs, eliminating the need for a separate cerebral CPG.

Our study represents one of the first demonstrations that dissimilar motor structures can be controlled by a single CPG. This simple mechanism may be effective because of the tight coupling between the activity of radula protraction and lip motoneurons. In fact, we argue that, among the examples of coordination between dissimilar structures listed earlier, Aplysia lip-radula coordination may entail the tightest coupling, i.e., the phasing of lip motoneuron activity closely matches that of radula protraction motoneuron activity. Consequently, phasing and cycle period 

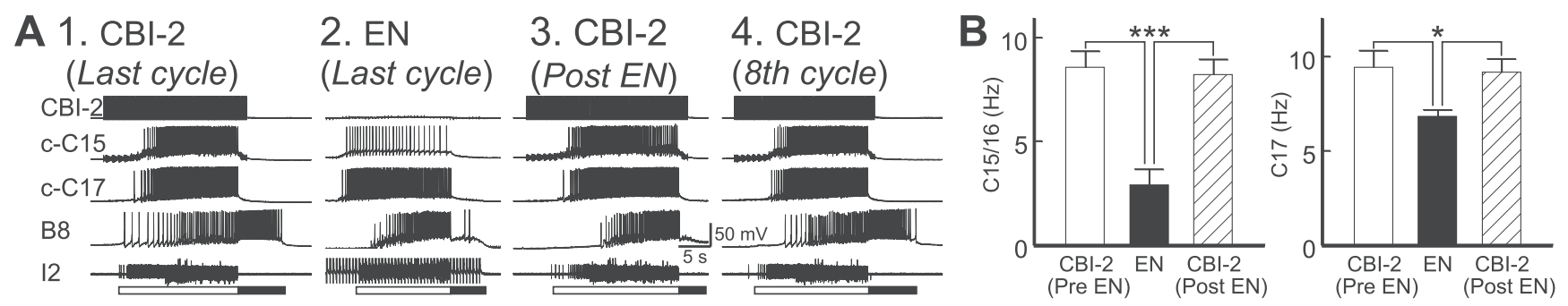

Figure 11. Activity of $\mathrm{C} 15 / 16$ and (17 in a biasing experiment. $A$, (BI-2 was stimulated to induce eight cycles of motor programs (interprogram interval of $30 \mathrm{~s}$ ), the last of which is shown in $\boldsymbol{A} 1$. B8 fired predominantly during retraction (filled bar); therefore, the program was ingestive. In this program, both C15 and C17 fired strongly. Subsequently the EN was stimulated for 5 min to elicit 10 cycles, the last of which is shown in A2. B8 fired primarily during protraction (open bar), indicating that the program was egestive. In this program, C15 fired weakly but C17 showed substantial activity. Following EN stimulation, CBI-2 was activated. The first program is shown in A3. This CBI-2-elicited program became egestive. In this program, both C15 and C17 fired strongly. CBI-2-evoked activity again became ingestive after eight more cycles (A4). B, Group data showing activity of $\mathrm{C} 15 / 16$ (left) or C17 (right). ${ }^{*} p<0.05,{ }^{* * *} p<0.001$. Error bars indicate SEM.
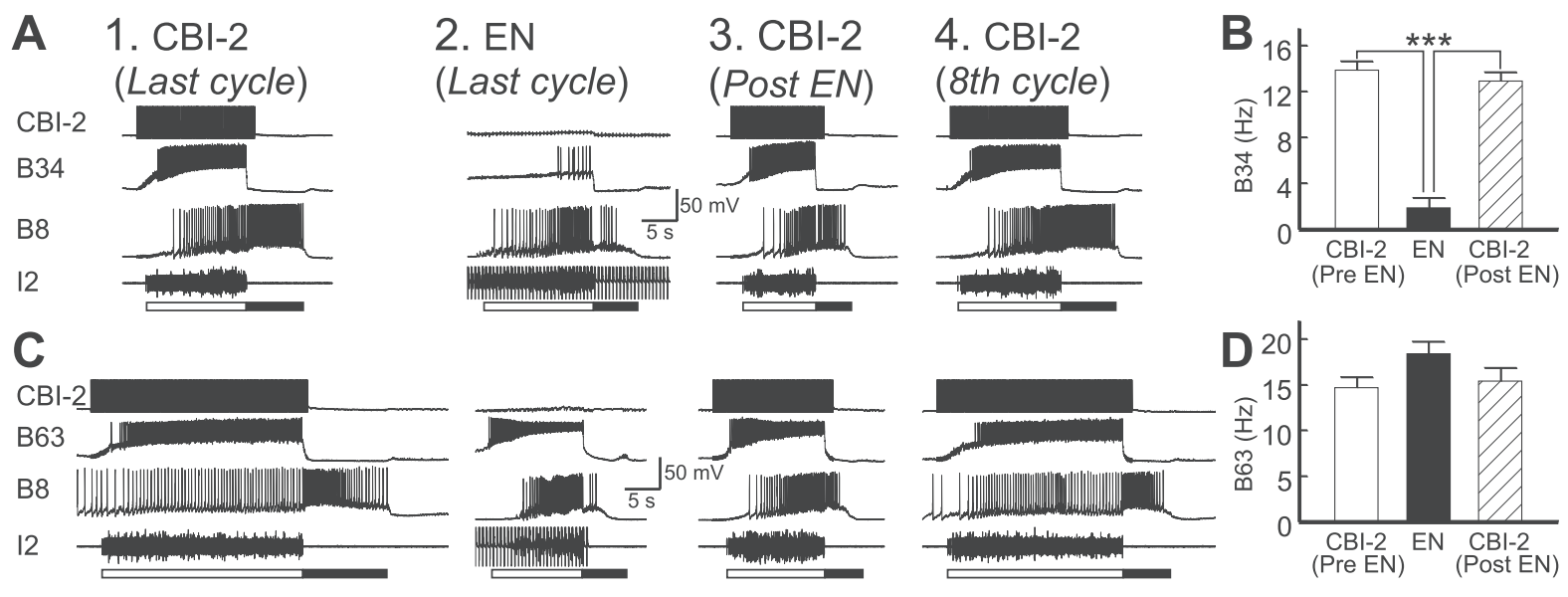

Figure 12. Activity of $B 34$ and $B 63$ in a biasing experiment similar to the one shown in Figure 11. $A$, Activity of $B 34$ during biasing. $B$, Group data showing $B 34$ activity. ${ }^{* * *} p<0.001$. $B 34$ was strongly active whenever (BI-2 was stimulated, but was only weakly active during EN stimulation. C, An example showing activity of B63 during biasing. D, Group data showing B63 activity. B63 was strongly active in programs elicited by either CBI-2 or EN. Error bars indicate SEM.

of the motoneurons controlling one structure (the lips) is primarily mediated by the CPG controlling the other structure (the radula).

In cases involving separate CPGs, synaptic interactions can be diverse and depend on the specific coordinating patterns. For example, crustacean pyloric and gastric mill circuits are mediated by two separate CPGs generating different cycle periods. In this intercircuit coordination, a single inhibitory synapse from the pyloric CPG to the gastric mill CPG is essential in determining the slower gastric mill cycle period (Nadim et al., 1998; Bartos et al., 1999). In segmental circuits, a gradient of excitation along multiple segments mediated by coordinating projection neurons has been proposed to mediate intersegmental phase differences of fish and leech swimming circuits (Williams, 1992; Mullins et al., 2011), and the leech heartbeat circuit (Hill et al., 2003). This type of control has recently been directly demonstrated in the crayfish swimmeret circuit (Smarandache et al., 2009).

\section{Generation of multiple forms of motor patterns in dissimilar motor structures}

One fundamental principle in motor control is that most motor networks are multifunctional (Nusbaum and Beenhakker, 2002; Jing and Weiss, 2005; Briggman and Kristan, 2008; Jing et al., 2009). Thus, the Aplysia buccal CPG produces two major types of motor programs: ingestive and egestive. This raises the question of how lip motoneuron activity is coordinated with radula motoneuron activity when the buccal CPG generates different motor outputs. This issue is of great interest since multifunctionality in the context of motor coordination is commonly observed, e.g., in the coordination of respiration and locomotion during normal activity versus exercise (Gariépy et al., 2010), but mechanisms are poorly understood.

In ingestive versus egestive programs that are elicited by CBI-2 versus $\mathrm{EN}$ respectively, although lip motoneurons are active during radula protraction, their activity levels do vary. To determine whether this variability results from the difference in nature of the programs (ingestive vs egestive) or the inputs used to evoke activity (CBI-2 vs EN), we used a previously established biasing paradigm (Proekt et al., 2004). In this situation, CBI-2 stimulation elicits an egestive program immediately following EN stimulation (Fig. 11). This revealed that C15/16 fired strongly in egestive programs when CBI-2 was used to trigger the program, suggesting that $\mathrm{C} 15 / 16$ activity is specific to CBI-2 input. These data imply that egestive programs may have two subtypes, one elicited by EN and one elicited by CBI-2, distinguished by C15/16 activity levels. Egestive programs elicited by EN serve to reject inedible food that is already in the buccal cavity (Chiel et al., 1986), and do not require strong opening of the mouth. Although we have not studied this directly, we speculate that egestive programs elicited by CBI-2 may serve to reject inedible food that is around the mouth, which may require a larger opening of the lips for the radula to access the food. More generally, our data suggest that it is critical to study multifunctionality in the context of movement coordination between dissimilar motor structures 
A

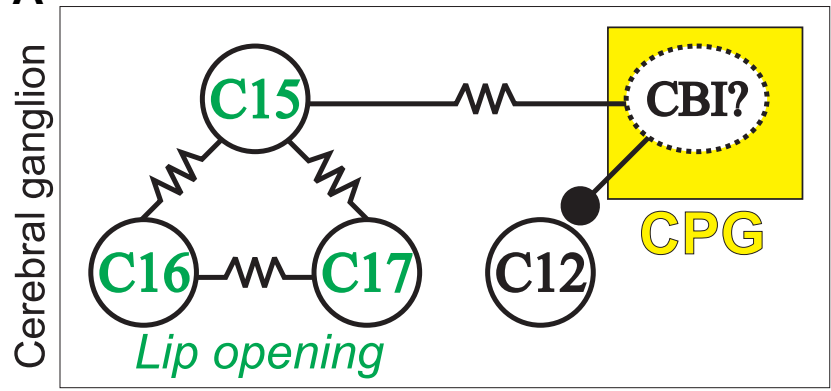

B

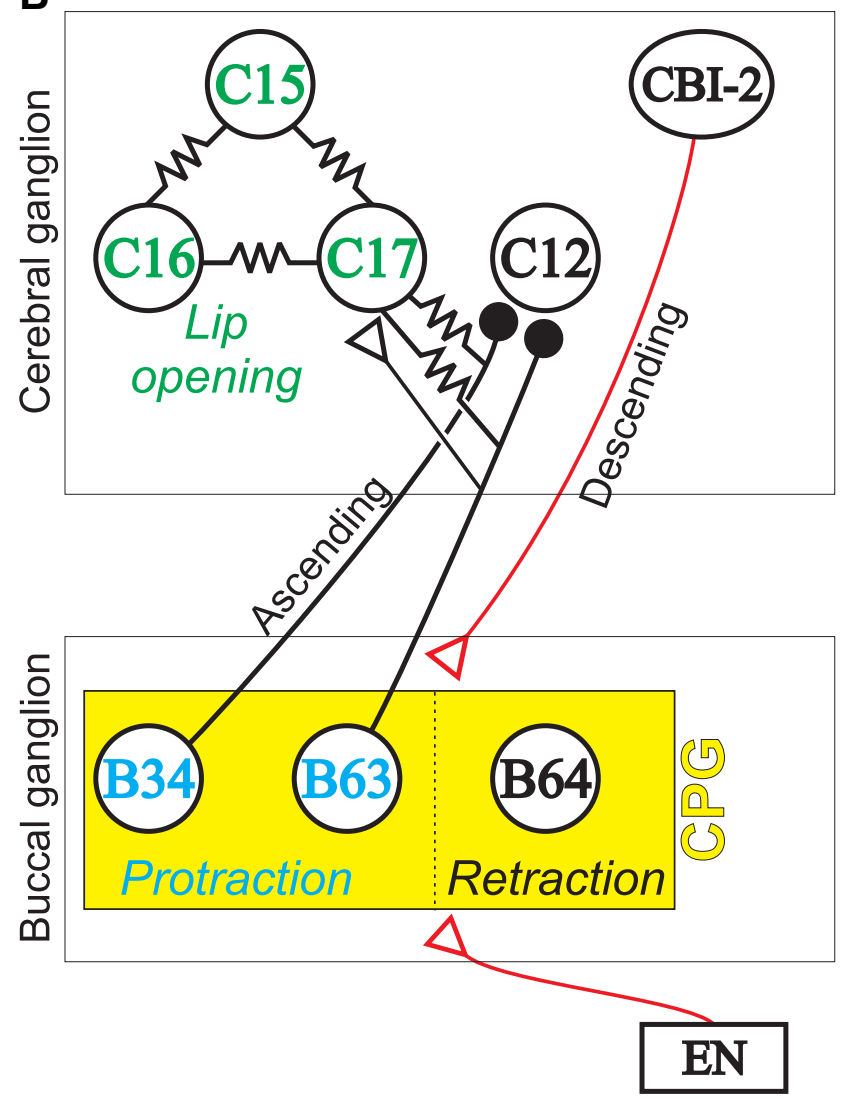

Figure 13. Circuitry diagrams of two $C P G$ s or a single $C P G$ that control the activity of lip motoneurons. A, The putative cerebral CPG as postulated by Perrins and Weiss (1996). C15, C16, and $C 17$ are electrically coupled (Perrins and Weiss, 1996). The critical element of the putative cerebral CPG was postulated to be a CBI(?) that is electrically coupled to C15/16/17, accounting for the ability of $D C$ stimulation of $\mathrm{C} 15$ to activate it, and thereby CMPs. Further, it was hypothesized that the CBI makes an inhibitory synaptic connections with C12. Thus, two CPGs, the cerebral CPG and buccal CPG (not depicted), would coordinate activity of lip motoneurons. $\boldsymbol{B}$, Neural circuit based on findings in this paper. There is a single buccal CPG controlling lip motoneuron activity. The cerebral axon of the buccal CPG element B34 is electrically coupled to $C 15 / 16 / 17$. Thus, the cerebral cyclic activity evoked by DC stimulation of $\mathrm{C} 15$ can be accounted for by the activation of B34 cerebral axons through electrical coupling. Moreover, we demonstrate an inhibitory synaptic connection between B34 and C12. B63 makes similar connections, but its IPSP to C 12 is smaller. Consequently B63 plays a lesser role in activation of CMPs. Nonetheless, because B34 and B63 provide substantial excitation of $(15 / 16 / 17$, both contribute to activity in these cells during the expression of feeding motor programs. Also illustrated are the two inputs (red) that activate the buccal CPG: (BI-2, which receives afferent inputs for head sensory organs regarding food, and the EN, which innervates the esophagus and the gut. Open triangle, excitation; closed circle, inhibition; resistor symbol, electrical coupling. as it may unravel novel ways to generate various motor tasks that meet diverse extrinsic and intrinsic demands.

The discovery of interneurons driving lip motoneurons also explains how different coordination patterns during various forms of programs can be implemented. Input-specific activity of C15/16 and program-shared activity of C17 are mediated by specific actions of B34 and B63. B34 preferentially excites lip motoneurons during $\mathrm{CBI}-2$ programs, while $\mathrm{B} 63$ provides the primary excitatory drive to C17 during EN programs. Activity patterns of B34 and B63 during ingestive versus egestive programs are consistent as B34 is primarily active during CBI-2 programs, while B63 exhibits substantial activity in all programs. Thus, B34 and B63 combine to mediate not only activity phasing of lip motoneurons, but also their distinct activity patterns during the expression of various motor programs.

\section{Activity coordination by ascending projection neurons: role} of electrical coupling

Most neural networks involve a local circuit (e.g., a CPG) that interacts with two types of projection neurons, which are the inputs to the circuit (e.g., a CBI) or the outputs of the circuit (e.g., a BCI) (Fig. 13B). Evidence has demonstrated growing importance of electrical coupling in generating network output. In invertebrate and vertebrate CPG networks as well as more complex cortical networks, electrical coupling plays a critical role in coordinating neuronal activity, e.g., promoting synchronized activity of local circuit neurons (Bennett and Zukin, 2004).

Our study highlights the critical role of electrical coupling in coordinating activity in remote brain areas by ascending projection neurons. Other examples of electrical coupling between projection neurons and remote target neurons include descending projection neurons in crayfish (Edwards et al., 1999) and crab (Coleman et al., 1995) and reticulospinal neurons in lamprey (Rovainen, 1979) and fish (Korn and Faber, 2005). Most of these cases involve mixed chemical and electrical synapses, similar to the B63 synapse with lip motoneurons. Purely electrical synapses such as B34 synapse with lip motoneurons have yet to be described in other systems. One notable feature of descending projection neurons is that they often act on diverse targets, with a subset of connections implemented by electrical coupling. Our data suggest that ascending projection neurons like B34 and B63 do so as well. Thus, transmitting information to diverse remote targets using both chemical and electrical synapses may be a common property of projection neurons.

In cortical circuits, it is presently unknown whether glutamatergic and GABAergic (Jinno et al., 2007) projection neurons form electrical synapses with neurons in remote brain regions. Such a possibility is, however, likely considering the presence of electrical coupling between GABAergic interneurons (Tamás et al., 2000; Galarreta and Hestrin, 2001) as well as between projection neurons (Schmitz et al., 2001). If such electrical coupling exists, it will be important to consider possible roles of electrically coupled axons in lesion experiments, at least shortly after the lesion. In this situation, local circuit neurons might still activate surviving axons through electrical coupling.

In summary, using various approaches, we showed how the closely coupled activity of motoneurons controlling two motor structures is implemented by a single CPG. Moreover, axodendritic electrical coupling by projection neurons represents a pivotal and novel part of the coupling mechanisms within the distributed CPG. Growing appreciation of the importance of electrical coupling in network function raises the possibility that 
similar mechanisms are used to coordinate activity in remotely localized neurons from other systems, including vertebrates.

\section{References}

Bartos M, Manor Y, Nadim F, Marder E, Nusbaum MP (1999) Coordination of fast and slow rhythmic neuronal circuits. J Neurosci 19:6650-6660.

Bennett MV, Zukin RS (2004) Electrical coupling and neuronal synchronization in the mammalian brain. Neuron 41:495-511.

Briggman KL, Kristan WB (2008) Multifunctional pattern-generating circuits. Annu Rev Neurosci 31:271-294.

Chiel HJ, Weiss KR, Kupfermann I (1986) An identified histaminergic neuron modulates feeding motor circuitry in Aplysia. J Neurosci 6:2427-2450.

Church PJ, Lloyd PE (1994) Activity of multiple identified motor neurons recorded intracellularly during evoked feedinglike motor programs in Aplysia. J Neurophysiol 72:1794-1809.

Coleman MJ, Meyrand P, Nusbaum MP (1995) A switch between two modes of synaptic transmission mediated by presynaptic inhibition. Nature 378:502-505.

Davis WJ, Kovac MP, Croll RP, Matera EM (1984) Brain oscillator(s) underlying rhythmic cerebral and buccal motor output in the mollusc, Pleurobranchaea californica. J Exp Biol 110:1-15.

Dembrow NC, Jing J, Brezina V, Weiss KR (2004) A specific synaptic pathway activates a conditional plateau potential underlying protraction phase in the Aplysia feeding central pattern generator. J Neurosci 24:5230-5238.

Dietz V (2002) Do human bipeds use quadrupedal coordination? Trends Neurosci 25:462-467.

Dietz V, Michel J (2009) Human bipeds use quadrupedal coordination during locomotion. Ann N Y Acad Sci 1164:97-103.

Edwards DH, Heitler WJ, Krasne FB (1999) Fifty years of a command neuron: the neurobiology of escape behavior in the crayfish. Trends Neurosci 22:153-161.

Evans CG, Jing J, Rosen SC, Cropper EC (2003) Regulation of spike initiation and propagation in an Aplysia sensory neuron: gating-in via central depolarization. J Neurosci 23:2920-2931.

Friedman AK, Zhurov Y, Ludwar BCh, Weiss KR (2009) Motor outputs in a multi-tasking network: relative contributions of inputs and experiencedependent states. J Neurophysiol 102:3711-3727.

Galarreta M, Hestrin S (2001) Electrical synapses between GABA-releasing interneurons. Nat Rev Neurosci 2:425-433.

Gariépy JF, Missaghi K, Dubuc R (2010) The interactions between locomotion and respiration. Prog Brain Res 187:173-188.

Grillner S (1981) Control of locomotion in bipeds, tetrapods, and fish. In: Handbook of physiology, Sec 1, Vol 2 (Brooks V, ed), pp 1179-1236. Bethesda, MD: American Physiological Society.

Grillner S (2006) Biological pattern generation: the cellular and computational logic of networks in motion. Neuron 52:751-766.

Harris-Warrick RM, Marder E, Selverston AI, Moulins M, eds (1992) Dynamic biological networks: the stomatogastric nervous system. Cambridge, MA: MIT Press.

Hill AA, Masino MA, Calabrese RL (2003) Intersegmental coordination of rhythmic motor patterns. J Neurophysiol 90:531-538.

Hurwitz I, Susswein AJ (1996) B64, a newly identified central pattern generator element producing a phase switch from protraction to retraction in buccal motor programs of Aplysia californica. J Neurophysiol 75:1327-1344.

Hurwitz I, Goldstein RS, Susswein AJ (1994) Compartmentalization of pattern-initiation and motor functions in the $\mathrm{B} 31$ and $\mathrm{B} 32$ neurons of the buccal ganglia of Aplysia californica. J Neurophysiol 71:1514-1527.

Hurwitz I, Kupfermann I, Susswein AJ (1997) Different roles of neurons B63 and B34 that are active during the protraction phase of buccal motor programs in Aplysia californica. J Neurophysiol 78:1305-1319.

Hurwitz I, Kupfermann I, Weiss KR (2003) Fast synaptic connections from CBIs to pattern-generating neurons in Aplysia: initiation and modification of motor programs. J Neurophysiol 89:2120-2136.

Jing J, Weiss KR (2001) Neural mechanisms of motor program switching in Aplysia. J Neurosci 21:7349-7362.

Jing J, Weiss KR (2002) Interneuronal basis of the generation of related but distinct motor programs in Aplysia: implications for current neu- ronal models of vertebrate intralimb coordination. J Neurosci $22: 6228-6238$

Jing J, Weiss KR (2005) Generation of variants of a motor act in a modular and hierarchical motor network. Curr Biol 15:1712-1721.

Jing J, Vilim FS, Wu JS, Park JH, Weiss KR (2003) Concerted GABAergic actions of Aplysia feeding interneurons in motor program specification. J Neurosci 23:5283-5294.

Jing J, Cropper EC, Hurwitz I, Weiss KR (2004) The construction of movement with behavior-specific and behavior-independent modules. J Neurosci 24:6315-6325.

Jing J, Vilim FS, Horn CC, Alexeeva V, Hatcher NG, Sasaki K, Yashina I, Zhurov Y, Kupfermann I, Sweedler JV, Weiss KR (2007) From hunger to satiety: reconfiguration of a feeding network by Aplysia neuropeptide Y. J Neurosci 27:3490-3502.

Jing J, Gillette R, Weiss KR (2009) Evolving concepts of arousal: insights from simple model systems. Rev Neurosci 20:405-427.

Jing J, Sweedler JV, Cropper EC, Alexeeva V, Park JH, Romanova EV, Xie F, Dembrow NC, Ludwar BC, Weiss KR, Vilim FS (2010) Feedforward compensation mediated by the central and peripheral actions of a single neuropeptide discovered using representational difference analysis. J Neurosci 30:16545-16558.

Jinno S, Klausberger T, Marton LF, Dalezios Y, Roberts JD, Fuentealba P, Bushong EA, Henze D, Buzsáki G, Somogyi P (2007) Neuronal diversity in GABAergic long-range projections from the hippocampus. J Neurosci 27:8790-8804

Korn H, Faber DS (2005) The Mauthner cell half a century later: a neurobiological model for decision-making? Neuron 47:13-28.

Kristan WB Jr, Calabrese RL, Friesen WO (2005) Neuronal control of leech behavior. Prog Neurobiol 76:279-327.

Ludwar BCh, Göritz ML, Schmidt J (2005) Intersegmental coordination of walking movements in stick insects. J Neurophysiol 93:1255-1265.

Morgan PT, Jing J, Vilim FS, Weiss KR (2002) Interneuronal and peptidergic control of motor pattern switching in Aplysia. J Neurophysiol 87:49-61.

Morton DW, Chiel HJ (1993a) In vivo buccal nerve activity that distinguishes ingestion from rejection can be used to predict behavioral transitions in Aplysia. J Comp Physiol A 172:17-32.

Morton DW, Chiel HJ (1993b) The timing of activity in motor neurons that produce radula movements distinguishes ingestion from rejection in $A p$ lysia. J Comp Physiol A 173:519-536.

Mullins OJ, Hackett JT, Buchanan JT, Friesen WO (2011) Neuronal control of swimming behavior: comparison of vertebrate and invertebrate model systems. Prog Neurobiol 93:244-269.

Nadim F, Manor Y, Nusbaum MP, Marder E (1998) Frequency regulation of a slow rhythm by a fast periodic input. J Neurosci 18:5053-5067.

Nargeot R, Baxter DA, Byrne JH (1997) Contingent-dependent enhancement of rhythmic motor patterns: an in vitro analog of operant conditioning. J Neurosci 17:8093-8105.

Nishikawa KC (1999) Neuromuscular control of prey capture in frogs. Philos Trans R Soc Lond B Biol Sci 354:941-954.

Nusbaum MP, Beenhakker MP (2002) A small-systems approach to motor pattern generation. Nature 417:343-350.

Perrins R, Weiss KR (1996) A cerebral central pattern generator in Aplysia and its connections with buccal feeding circuitry. J Neurosci 16:7030-7045.

Perrins R, Weiss KR (1998) Compartmentalization of information processing in an Aplysia feeding circuit interneuron through membrane properties and synaptic interactions. J Neurosci 18:3977-3989.

Proekt A, Brezina V, Weiss KR (2004) Dynamical basis of intentions and expectations in a simple neuronal network. Proc Natl Acad Sci U S A 101:9447-9452.

Proekt A, Jing J, Weiss KR (2007) Multiple contributions of an inputrepresenting neuron to the dynamics of the Aplysia feeding network. J Neurophysiol 97:3046-3056.

Rosen SC, Teyke T, Miller MW, Weiss KR, Kupfermann I (1991) Identification and characterization of cerebral-to-buccal interneurons implicated in the control of motor programs associated with feeding in Aplysia. J Neurosci 11:3630-3655.

Rovainen CM (1979) Neurobiology of lampreys. Physiol Rev 59:1007-1077.

Sasaki K, Brezina V, Weiss KR, Jing J (2009) Distinct inhibitory neurons exert temporally specific control over activity of a motoneuron receiving concurrent excitation and inhibition. J Neurosci 29:11732-11744. 
Schmitz D, Schuchmann S, Fisahn A, Draguhn A, Buhl EH, Petrasch-Parwez E, Dermietzel R, Heinemann U, Traub RD (2001) Axo-axonal coupling: a novel mechanism for ultrafast neuronal communication. Neuron $31: 831-840$

Smarandache C, Hall WM, Mulloney B (2009) Coordination of rhythmic motor activity by gradients of synaptic strength in a neural circuit that couples modular neural oscillators. J Neurosci 29:9351-9360.

Susswein AJ, Byrne JH (1988) Identification and characterization of neurons initiating patterned neural activity in the buccal ganglia of Aplysia. J Neurosci 8:2049-2061.

Tamás G, Buhl EH, Lörincz A, Somogyi P (2000) Proximally targeted GABAergic synapses and gap junctions synchronize cortical interneurons. Nat Neurosci 3:366-371.
Teyke T, Rosen SC, Weiss KR, Kupfermann I (1993) Dopaminergic neuronB20 generates rhythmic neuronal activity in the feeding motor circuitry of Aplysia. Brain Res 630:226-237.

Weiss KR, Kupfermann I (1976) Homology of the giant serotonergic neurons (metacerebral cells) in Aplysia and pulmonate molluscs. Brain Res 117:33-49.

Williams TL (1992) Phase coupling by synaptic spread in chains of coupled neuronal oscillators. Science 258:662-665.

Wu JS, Vilim FS, Hatcher NG, Due MR, Sweedler JV, Weiss KR, Jing J (2010) Composite modulatory feedforward loop contributes to the establishment of a network state. J Neurophysiol 103:2174-2184.

Zehr EP, Duysens J (2004) Regulation of arm and leg movement during human locomotion. Neuroscientist 10:347-361. 